\title{
CO Tip Functionalization Inverts Atomic Force Microscopy Contrast via Short-Range Electrostatic Forces
}

\author{
Maximilian Schneiderbauer, Matthias Emmrich, Alfred J. Weymouth, and Franz J. Giessibl \\ Institute of Experimental and Applied Physics, University of Regensburg, 93040 Regensburg, Germany
} (Received 20 February 2014; published 23 April 2014)

\begin{abstract}
We investigate insulating $\mathrm{Cu}_{2} \mathrm{~N}$ islands grown on $\mathrm{Cu}(100)$ by means of combined scanning tunneling microscopy and atomic force microscopy with two vastly different tips: a bare metal tip and a CO-terminated tip. We use scanning tunneling microscopy data as proposed by Choi, Ruggiero, and Gupta to unambiguously identify atomic positions. Atomic force microscopy images taken with the two different tips show an inverted contrast over $\mathrm{Cu}_{2} \mathrm{~N}$. The observed force contrast can be explained with an electrostatic model, where the two tips have dipole moments of opposite directions. This highlights the importance of short-range electrostatic forces in the formation of atomic contrast on polar surfaces in noncontact atomic force microscopy.
\end{abstract}

DOI: 10.1103/PhysRevLett.112.166102

PACS numbers: 68.37.Ps, 61.46.-w, 68.37.Ef

The combination of scanning tunneling microscopy (STM) with noncontact atomic force microscopy (NC-AFM) in a single probe enables a wide range of atomic-scale studies on surfaces. Whereas contrast mechanisms in STM for different tip-sample systems are widely understood, the interpretation of NC-AFM data remains challenging. In NC-AFM the sum over all tip-sample interactions is measured, and the source of atomic resolution is often hard to identify. On semiconductors [1] —as well as on metals [2] —imaged with reactive tips (e.g., Si) atomic contrast is dominated by the formation of covalent bonds that often reach magnitudes of nanonewtons. For nonreactive CO-functionalized tips, Pauli repulsion was attributed to the observed intramolecular resolution $[3,4]$. Lantz et al. [5] showed that the dangling bonds of $\mathrm{Si}(111)-(7 \times 7)$ can induce a dipole moment in (nonreactive) oxidized Si tips resulting in a short-range electrostatic interaction, which contributes to atomic resolution. Electrostatic interaction and an induced tip dipole moment was also used to explain atomic contrast on ionic crystals [6]. A similar model describes the interaction with charged adatoms on thin insulating layers $[7,8]$. Moreover, it was found that clean metallic tips carry an intrinsic dipole moment $[9,10]$, which is caused by the Smoluchowski effect [11]. All of these examples underline the importance of atomic-scale electrostatic interactions in NC-AFM.

Electrostatic forces become even more meaningful as polar thin insulating layers (e.g., $\mathrm{NaCl}, \mathrm{MgO}, \mathrm{Cu}_{2} \mathrm{~N}$ ) are used to decouple adsorbates in STM and AFM experiments $[3,7,12-16]$. In this study we explore the influence of electrostatic forces in NC-AFM on $\mathrm{Cu}_{2} \mathrm{~N}$ islands on $\mathrm{Cu}(100)$. $\mathrm{N}$ and $\mathrm{Cu}$ atoms on $\mathrm{Cu}_{2} \mathrm{~N}$ form a periodic charge arrangement, as calculated by density functional theory (DFT) [17] [Figs. 1(c)-1(e)]. Compared to alkali halides, the $\mathrm{Cu}_{2} \mathrm{~N}$ 's $c(2 \times 2)$ unit cell structure has a lower symmetry; thus, its atomic positions are easier to designate.
STM experiments led to two criteria to locate $\mathrm{N}$ atoms within the islands [18]: first, $\mathrm{N}$ adsorbs on the hollow sites of $\mathrm{Cu}(100)$ [19-21] and should therefore appear fourfold symmetric; second, island boundaries and sharp edges are determined by $\mathrm{N}$ atoms [15]. With this, $\mathrm{N}-, \mathrm{Cu}-$, and hollow sites can be identified in the $\mathrm{Cu}_{2} \mathrm{~N}$ unit cell [Fig. 1(c)].

In this Letter, we report on high-resolution simultaneously recorded current and force data of the $\mathrm{Cu}_{2} \mathrm{~N}$ surface. We compare interaction forces probed with a

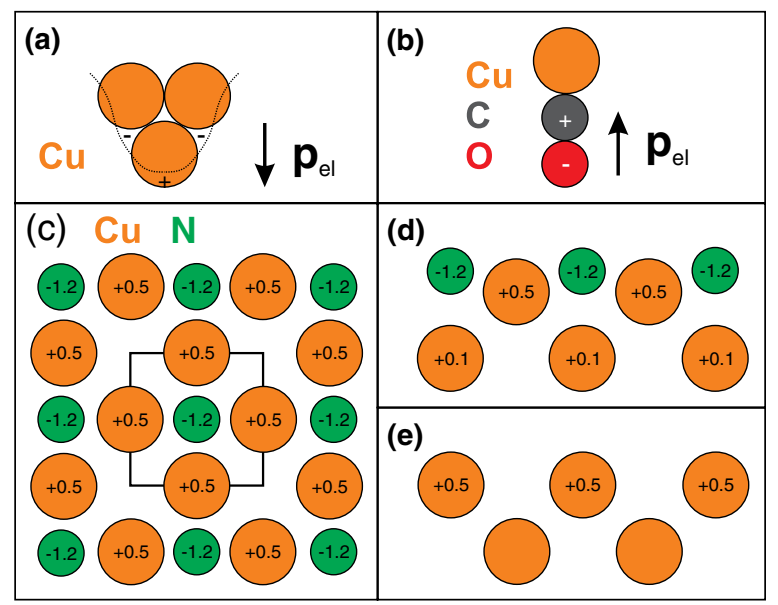

FIG. 1 (color online). (a) Charge redistribution in a metallic tip due to the Smoluchowski effect, leading to an electric dipole moment pointing towards the sample. (b) A CO molecule adsorbed on a copper tip, carrying an electric dipole moment pointing away from the sample. (c) Model of the $\mathrm{Cu}_{2} \mathrm{~N}$ network on $\mathrm{Cu}(100)$ with the numbers being the net charge of $\mathrm{Cu}$ and $\mathrm{N}$ atoms in units of elementary charge $e$ [17]. (d) Cross section of $\mathrm{Cu}_{2} \mathrm{~N}$ along a $\mathrm{N}-\mathrm{Cu}-\mathrm{N}$ row and (e) Cu-hollow site- $\mathrm{Cu}$ row showing the first two atomic layers of the reconstructed surface and the net charges of the atoms, respectively. In (c), (d), and (e), larger circles indicate $\mathrm{Cu}$ and smaller circles indicate $\mathrm{N}$. 
CO-terminated tip to data acquired with a metal tip. The force contrast, though, is inverted. We propose an electrostatic model where the two tips have opposite dipole moments. The calculated force contrast within a $\mathrm{Cu}_{2} \mathrm{~N}$ unit cell provides good agreement to the data.

All experiments were carried out with a home-built lowtemperature system at $6 \mathrm{~K}$ using a qPlus sensor $\left(f_{0}=29098 \mathrm{~Hz}, k=1800 \mathrm{~N} \mathrm{~m}^{-1}\right)$ [22] equipped with a $\mathrm{W}$ tip, operated with small amplitudes $(50 \mathrm{pm})$ in frequency modulation mode. Metallic $(\mathrm{Cu})$ tips were prepared by strongly poking them into a clean $\mathrm{Cu}$ sample while applying $200 \mathrm{~V}$. Tips were functionalized with $\mathrm{CO}$ molecules following the standard procedure [23]. $\mathrm{Cu}_{2} \mathrm{~N}$ islands were prepared by sputtering a clean $\mathrm{Cu}(100)$ crystal with $\mathrm{N}$ gas for $120 \mathrm{~s}$ and heating it to $600 \mathrm{~K}$ for $300 \mathrm{~s}$. Forces were calculated by applying the Sader-Jarvis-deconvolution method [24] to our recorded three-dimensional frequency shift maps.

Figure 2(a) shows a STM topograph of an island using a $\mathrm{Cu}$-terminated tip recorded with a set point of $500 \mathrm{pA}$ at a tip voltage of $10 \mathrm{mV}$. The image was Laplace filtered and inverted (Laplace filtering inverts brightness) to enhance contrast [15] and a structural model is partially overlaid. The raw data of Fig. 2 can be found in the Supplemental Material [25].

When the $\mathrm{Cu}$ tip is terminated by a $\mathrm{CO}$ molecule, the STM contrast is different. The constant-current topograph in Fig. 2(d) was recorded using a set point of $100 \mathrm{pA}$ at a tip voltage of $10 \mathrm{mV}$. Three distinct features appear within the island: elongated protrusions, wide depressions, and - most remarkably - very narrow depressions. It is known that the strong $p$-wave character of CO-terminated tips influences the imaging contrast in STM [26]. In Ref. [26], the authors investigated the organic molecule pentacene, whose orbitals locally exhibit $\sigma, \pi$, and $\delta$ symmetry. They used Chen's derivative rule [27] to explain that due to the CO's $\pi$ orbital the tunneling matrix element turns to zero over regions where the pentacene's orbital resembles one with $\sigma$ and $\delta$ symmetries, whereas it does not where the orbital locally has $\pi$ symmetry. We propose a similar tunneling contrast formation for copper nitride, motivated by the DFT calculations of Soon et al. [21]. For the eigenstate closest to our bias voltage of $10 \mathrm{mV}, \mathrm{N}$ and $\mathrm{Cu}$ atoms show a local $\sigma$ character, in which the $\mathrm{N}$ wave function has twice the spatial extension of $\mathrm{Cu}$. Because of the $3 d$ state of $\mathrm{Cu}$ and the $2 p$ state of $\mathrm{N}$, the space in between $\mathrm{N}$ and $\mathrm{Cu}$ shows a local $\pi$ character. Taking into account the $\mathrm{N}-\mathrm{Cu}$ binding length of $183 \mathrm{pm}$, which is comparable to atomic distances within pentacene, we argue as follows: $\mathrm{N}$ exhibits a local $\sigma$ character and therefore the matrix element concerning the CO's $\pi$ orbital is zero, resulting in a very confined depression over $\mathrm{N}$. Between $\mathrm{N}$ and $\mathrm{Cu}$ the $\mathrm{CO}$ tip probes a local $\pi$ orbital enabling a tunneling current. $\mathrm{Cu}$ also has local $\sigma$ character, but its wave function only extends half as far compared to N. Furthermore, $\mathrm{Cu}$ is located $21 \mathrm{pm}$ lower
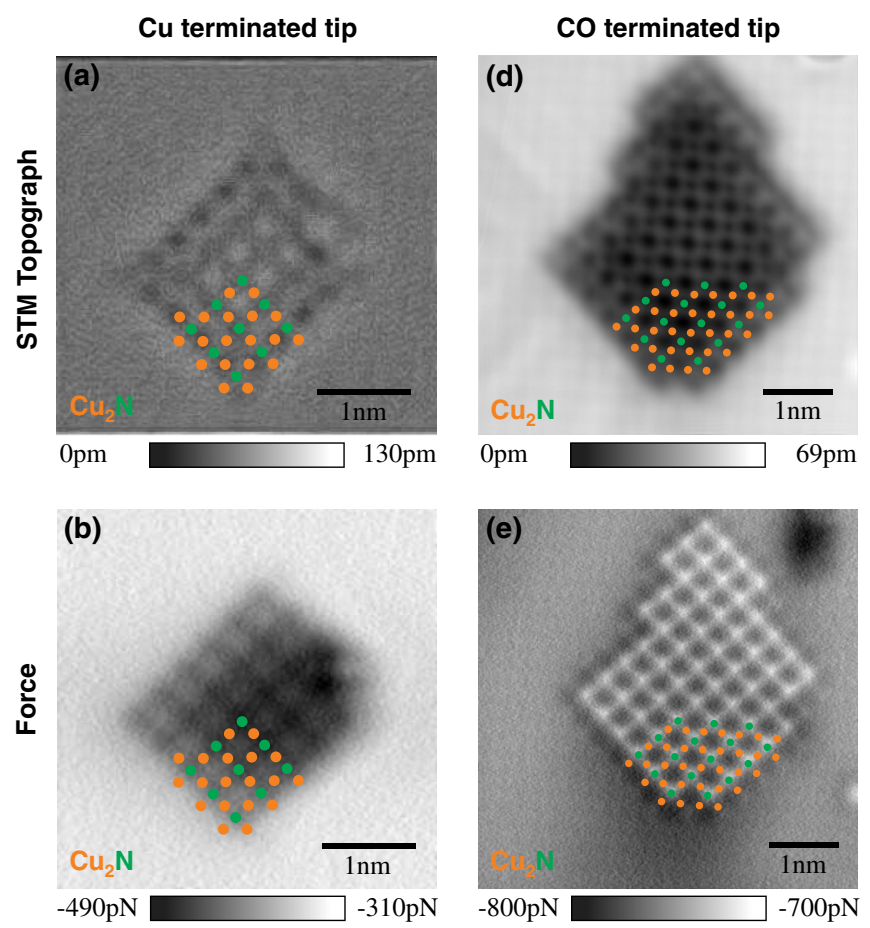

(c) $\Delta F(\mathrm{pN})$
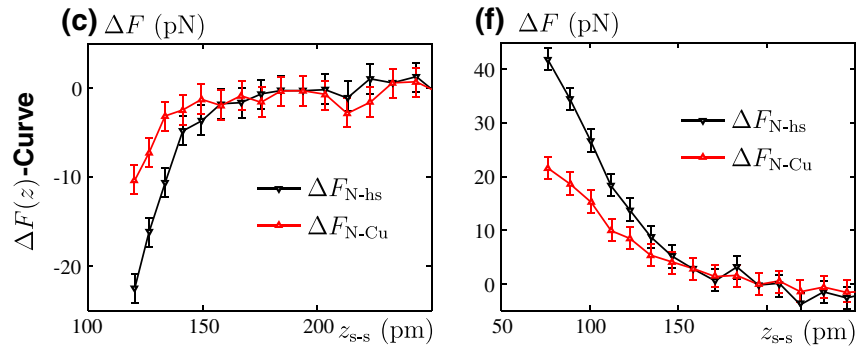

FIG. 2 (color online). Data acquired with a Cu-terminated tip [(a)-(c)] and with a CO-terminated tip [(d)-(f)]. Top row: STM topographs with the structural model of $\mathrm{Cu}_{2} \mathrm{~N}$ overlaid. Lighter circles indicate $\mathrm{Cu}$, darker circles indicate $\mathrm{N}$ : (a) is recorded at constant current at $500 \mathrm{pA}$ and $10 \mathrm{mV}$ applied to the tip and Laplace filtered to enhance contrast; (b) $100 \mathrm{pA}$ and $10 \mathrm{mV}$, raw data. Center row: Force between tip and sample at closest approach. Bottom row: Force contrast vs distance curves at two high-symmetry locations.

than $\mathrm{N}[20,21]$. From this we conclude that our microscope setup is not able to laterally resolve a depression over $\mathrm{Cu}$ as well, resulting in elongated protrusions centered over $\mathrm{Cu}$. For hollow sites, the lateral orbital overlap between tip and sample states is insufficient to yield a nonzero tunneling matrix element, leading to a wide depression. This lattice assignment fulfills the two required assignment criteria [Figs. 2(d)-2(e)].

Figure 2(b) shows the total force for a Cu-terminated tip at closest approach, corresponding to a STM set point of $28 \mathrm{nA}$ at a tip voltage of $10 \mathrm{mV}$ over bare $\mathrm{Cu}(100)$. Here, tip-sample distance is defined as the distance between the outermost atomic shells of the involved atoms (see Supplemental Material [25]), in the following called $z_{\mathrm{s}-\mathrm{s}}$. 
Using this definition of distance, Fig. 2(b) was measured at $z_{\mathrm{s}-\mathrm{s}}=125 \mathrm{pm}$ over bare $\mathrm{Cu}(100)$.

According to the lattice assignment for metal tips, $\mathrm{N}$ sites appear most attractive, followed by $\mathrm{Cu}$ sites and hollow sites. The measured overall force is attractive, because of the long-range van der Waals interaction, which normally does not depend on lateral position and thus does not allow for atomic resolution. Subtracting the forces between distinct unit-cell positions cancels out constant long-range interactions and the resulting force contrast contains site-dependent short-range components only. We define $\Delta F_{\mathrm{N}-\mathrm{Cu}}=F_{\mathrm{N} \text { site }}-F_{\mathrm{Cu} \text { site }}$ and $\Delta F_{\mathrm{N}-\mathrm{hs}}=F_{\mathrm{N} \text { site }}-F_{\mathrm{hs}}$ as the force difference (force contrast) between $\mathrm{N}$ and $\mathrm{Cu}$ sites and between $\mathrm{N}$ sites and hollow sites (hs). Table I shows the averaged force contrast for five different islands probed with different $\mathrm{Cu}$-terminated tips at similar tipsample distances. The short-range character of the interaction causing atomic contrast is reflected in the $\Delta F(z)$ curves displayed in Fig. 2(c). The atomic contrast is maintained over a vertical range of about $100 \mathrm{pm}$.

Figure 2(e) shows the total force at closest approach for a CO-terminated tip, which was at a STM set point of $3 \mathrm{nA}$ at $10 \mathrm{mV}$ over clean $\mathrm{Cu}(100)$. To determine the tip-sample distance the above-described model has to be adapted by using the point conductance of a $\mathrm{CO}$ molecule on the $\mathrm{Cu}$ surface. Experimentally, we find $G_{0}=(404,497 \Omega)^{-1}$ (see Supplemental Material [25]) and obtain $z_{\mathrm{s}-\mathrm{s}}=80 \mathrm{pm}$ for the closest approach. Figure 2(e) reveals an inverted force contrast compared to Fig. 2(b), with $\mathrm{N}$ atoms having the most repulsive interactions and hollow sites the most attractive interactions. The averaged force contrast for three CO-functionalized tips over three different islands at similar tip-sample distances is depicted in Table I. Figure 2(f) displays the above-defined $\Delta F(z)$ curves, showing repulsive force contrast (i.e., the force difference is positive) for approximately $100 \mathrm{pm}$.

The AFM images in the center row of Fig. 2 show a contrast inversion for $\mathrm{Cu}$ vs $\mathrm{CO}$ tips. We attribute this contrast inversion to opposite dipole moments of $\mathrm{Cu}$ - vs CO-terminated tips for two reasons. First, $\mathrm{Cu}_{2} \mathrm{~N}$ is a periodic arrangement of charged atoms. The electrostatic potential of such a periodic charge distribution with lattice constant $a$ decays exponentially [28], with a decay length given by $\lambda=a / 2 \pi\left(\mathrm{Cu}_{2} \mathrm{~N}: a=372 \mathrm{pm}\right.$ [18] and thus $\lambda=59 \mathrm{pm})$. This small decay length explains the shortrange character of this electrostatic interaction [Figs. 2(c) and 2(f)]. Second, in the following we will present an electrostatic model using a point-charge representation of the sample and tip. Using the force contrast and calculated tip-sample distances from the experiment, we fit the tip's dipole moment to model the experimental force dependence.

From experimental [18] as well as theoretical work $[17,20,21]$, the structural parameters and net charges of the $\mathrm{Cu}_{2} \mathrm{~N}$ 's unit-cell atoms are known. The distance between $\mathrm{N}$ and $\mathrm{Cu}$ is $183 \mathrm{pm}$, the $\mathrm{N}$ atoms are vertically displaced by $21 \mathrm{pm}$ with respect to the $\mathrm{Cu}$ surface layer, and the charged subsurface $\mathrm{Cu}$ layer has a distance of $197 \mathrm{pm}$ to the $\mathrm{Cu}$ surface layer, as indicated in Figs. 1(c)-1(e). Charges were calculated by DFT in Ref. [17] and are $-1.2 e$ for $\mathrm{N}$ atoms, $+0.5 e$ for surface $\mathrm{Cu}$ atoms, and $+0.1 e$ for the subsurface $\mathrm{Cu}$ atoms [Figs. 1(c)-1(e)]. Using these numbers, we constructed a three-dimensional point-charge representation of the sample with a size of $7 \times 7$ unit cells, including the subsurface $\mathrm{Cu}$ atoms, where all charges are located at the atomic-core positions. The resulting electric field was then used to calculate the interaction force with the tip (see Supplemental Material [25]). The Smoluchowski effect [11] causes a charge redistribution on corrugated metal surfaces, which leads to the formation of electric dipoles. For metallic tips, an electric dipole moment is formed pointing towards the sample [9], as depicted in Fig. 1(a). For a CO molecule adsorbed on a metal tip, the electric dipole moment, compared to the gas phase, changes in magnitude and sign due to charge transfer to the metal. We recently found experimental $[29,30]$ evidence that the CO's dipole moment is pointing into the metal, as depicted in Fig. 1(b), which is also supported by some ab initio molecular orbital calculations [31]. The tip was modeled as a simple dipole having two charges $\pm q$ separated by the dipole distance $d$.

For the $\mathrm{Cu}$-terminated tip, we got the best agreement with a charge of $\pm 0.13 e$ and $d=135 \mathrm{pm}$ (close to the atomic radius of $\mathrm{Cu}$ ) resulting in a dipole moment of $0.182 e \AA=0.874 \mathrm{D}$. The dipole points towards the sample, as described before, where the positive charge is located at the core of the front-most $\mathrm{Cu}$ tip atom. For the calculation, the positive tip charge ( $\mathrm{Cu}$ core) was placed at a distance of $395 \mathrm{pm}$ over the charges (cores) of the $\mathrm{Cu}$ surface layer. This distance was obtained by adding twice the atomic radius of $\mathrm{Cu}$ to the experimental $z_{\mathrm{s}-\mathrm{s}}=125 \mathrm{pm}$ (see Supplemental Material [25]). Figure 3(b) shows the calculated force between this metal tip dipole and all surface atoms. The force above the $\mathrm{Cu}$ site was set to zero

TABLE I. Comparison of averaged experimental and simulated force contrast for $\mathrm{Cu}$ - and $\mathrm{CO}$-terminated tips.

\begin{tabular}{|c|c|c|c|c|}
\hline & \multicolumn{2}{|c|}{$\Delta F_{\mathrm{N}-\mathrm{Cu}}$} & \multicolumn{2}{|c|}{$\Delta F_{\mathrm{N}-\mathrm{hs}}$} \\
\hline & Experiment & Simulation & Experiment & Simulation \\
\hline $\begin{array}{l}\text { Cu tip } \\
\text { CO tip }\end{array}$ & $\begin{array}{c}(-10 \pm 2) \mathrm{pN} \\
(22 \pm 1) \mathrm{pN}\end{array}$ & $\begin{array}{c}-12 \mathrm{pN} \\
26 \mathrm{pN}\end{array}$ & $\begin{array}{c}(-17 \pm 3) \mathrm{pN} \\
(39 \pm 2) \mathrm{pN}\end{array}$ & $\begin{array}{c}-17 \mathrm{pN} \\
34 \mathrm{pN}\end{array}$ \\
\hline
\end{tabular}



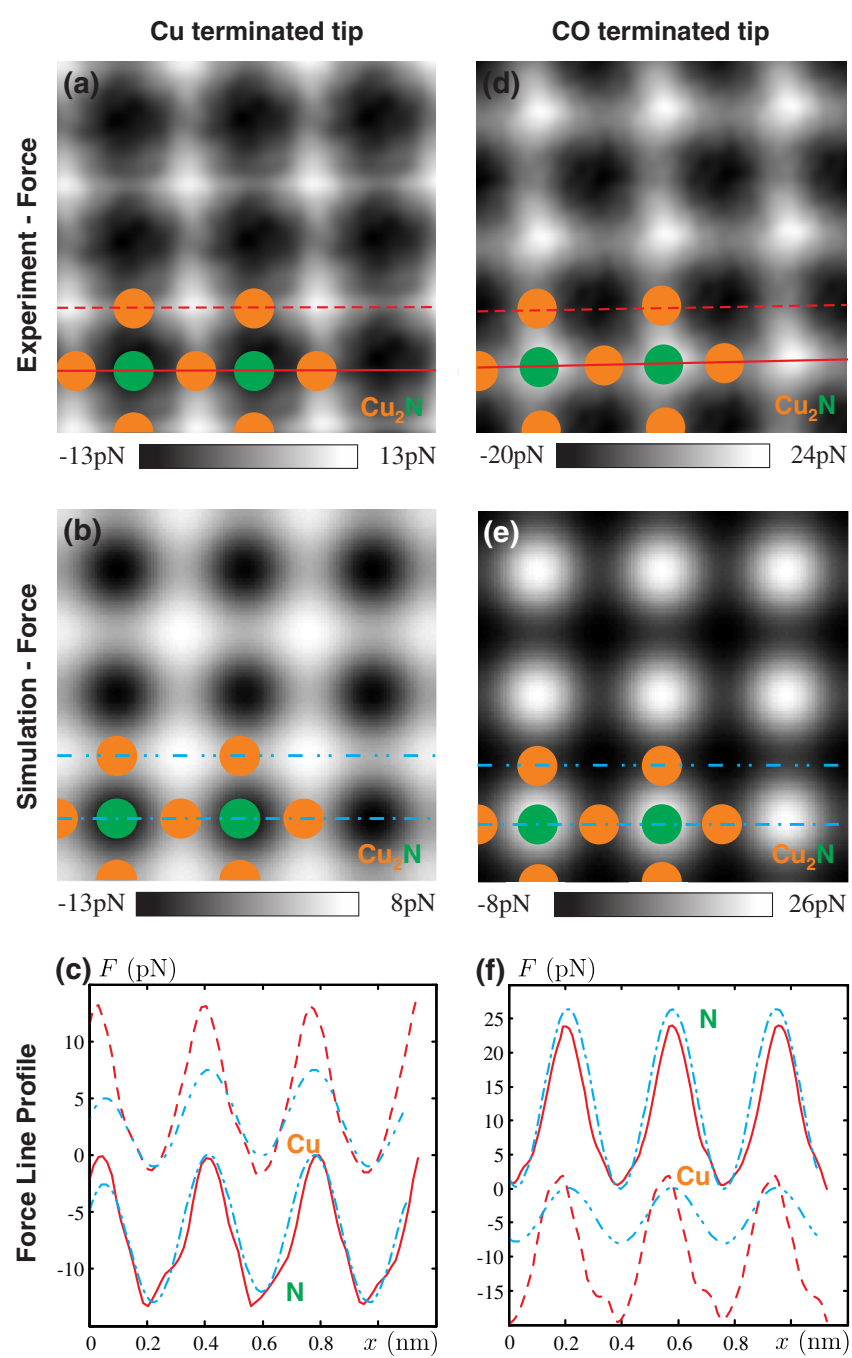

FIG. 3 (color online). Top row: Central area of Figs. 2(b) and 2(e) (unit-cell averaged and $3 \times 3$ Gauss filtered). Center row: Calculated electrostatic force between the tip dipole and the point charges of $\mathrm{a} \mathrm{Cu}_{2} \mathrm{~N}$ structure, including the second-layer $\mathrm{Cu}$ atoms (see also Fig. 1). Simulation for a $\mathrm{Cu}$-terminated tip with a dipole moment of $0.874 \mathrm{D}$ (b) and a CO-terminated tip with $0.035 \mathrm{D}$ (e). In the first two rows, the force at the $\mathrm{Cu}$ site was set to zero to emphasize the force difference. Bottom row: Line profiles from (a), (b), (d), and (e) along $\mathrm{N}-\mathrm{Cu}-\mathrm{N}$ and $\mathrm{Cu}$-hollow site-Cu directions, respectively.

to emphasize the force contrast. Line profiles along the $\mathrm{N}-\mathrm{Cu}-\mathrm{N}$ and $\mathrm{Cu}$-hollow site- $\mathrm{Cu}$ directions from Fig. 3(b) are plotted in Fig. 3(c) (dash-dotted line and dash-dot-dotted line). The relative interaction contrast is given in Table I and fits well to the experimental results. To compare the simulation to the experiment, Fig. 3(a) shows a zoom-in of the island in Fig. 2(b) (unit-cell average over the inner part plus $3 \times 3$ Gauss filter) with the respective line profiles in Fig. 3(c) (solid line and dashed line).

We modeled the $\mathrm{CO}$ tip as a dipole pointing away from the sample, where the negative charge sits at the $\mathrm{O}$ core and the positive charge at the $\mathrm{C}$ core. With the $\mathrm{CO}$ binding length of $115 \mathrm{pm}$ as the dipole distance $d$ and a charge of $\pm 0.03 e$, the dipole moment is $0.035 e \AA=0.166 \mathrm{D}$. The distance of the $\mathrm{O}$ core to the charges of the $\mathrm{Cu}$ surface layer was $275 \mathrm{pm}\left(z_{\mathrm{s}-\mathrm{s}}=80 \mathrm{pm}\right.$ plus the atomic radii of $\mathrm{O}, 60$ pm, and $\mathrm{Cu}, 135 \mathrm{pm}$ ) (see Supplemental Material [25]). In Fig. 3(e), the calculated force is shown with the force at the $\mathrm{Cu}$ site set to zero. Corresponding line profiles along the two prominent directions are given in Fig. 3(f) (dash-dotted line and dash-dot-dotted line). This simulation also gives quantitative agreement to our experimental force contrast (Table I). For comparison, Fig. 3(d) shows a zoom-in of Fig. 2(e) (unit-cell average over the inner part plus $3 \times 3$ Gauss filter) with the corresponding line profiles shown in Fig. 3(f) (solid line and dashed line).

This straightforward electrostatic model reproduces the relative force contrast of both tip terminations very well. The experimentally determined dipole moment of the $\mathrm{Cu}$ terminated tip of $0.874 \mathrm{D}$ is close to the range of previous work for $\mathrm{Cr}$ and $\mathrm{W}$ tips $[9,10]$. Simulation and experiment for the CO-terminated tip agree with the theory that the dipole moment points away from the sample. In general, the CO's dipole magnitude depends on the chemical nature and geometric structure of the adsorbent [31]. Hence, it is not surprising that the fit value of $0.166 \mathrm{D}$ varies from that of CO molecules adsorbed on surfaces [29,30,32].

The simulation does not account for (attractive) covalent bonds ( $\mathrm{Cu}$ tips) and Pauli repulsion ( $\mathrm{CO}$ tips). Both interactions are probably included in the measured overall force and would influence the force contrast and therefore the modeled dipole moment. However, the experimental core-core distances are just a lower boundary (Cu tip: 395 pm; CO tip: $275 \mathrm{pm}$ ) and are so large that we do not expect a strong contribution from either covalent bonds or Pauli repulsion.

In conclusion, we have shown that atomic resolution on relatively inert surfaces such as $\mathrm{Cu}_{2} \mathrm{~N}$ can be obtained by electrostatic multipole forces and not just by covalent bonding forces or Pauli repulsion forces. Richard Feynman already pointed out in his 1939 paper [33] that the force on a nucleus in an atomic system ultimately has an electrostatic origin. The difference we observed here compared to the covalent case in atomic imaging of semiconductors is that covalent bonds usually result in massive rearrangements of electronic charge density and subsequently forces in the nanonewton regime, while the forces here are only tens of piconewtons with much less redistribution of electronic charge.

The authors thank the Deutsche Forschungsgemeinschaft for funding within the SFB 689.

*maximilian.schneiderbauer@ur.de

[1] R. Pérez, M. C. Payne, I. Štich, and K. Terakura, Phys. Rev. Lett. 78, 678 (1997). 
[2] P. Dieška, I. Štich, and R. Pérez, Phys. Rev. Lett. 91, 216401 (2003).

[3] L. Gross, F. Mohn, N. Moll, P. Liljeroth, and G. Meyer, Science 325, 1110 (2009).

[4] N. Moll, L. Gross, F. Mohn, A. Curioni, and G. Meyer, New J. Phys. 12, 125020 (2010).

[5] M. A. Lantz, H. J. Hug, R. Hoffmann, S. Martin, A. Baratoff, and H.-J. Güntherodt, Phys. Rev. B 68, 035324 (2003).

[6] F. J. Giessibl, Phys. Rev. B 45, 13815 (1992).

[7] L. Gross, F. Mohn, P. Liljeroth, J. Repp, F. J. Giessibl, and G. Meyer, Science 324, 1428 (2009).

[8] F. Bocquet, L. Nony, and C. Loppacher, Phys. Rev. B 83, 035411 (2011).

[9] G. Teobaldi, K. Lämmle, T. Trevethan, M. Watkins, A. Schwarz, R. Wiesendanger, and A. L. Shluger, Phys. Rev. Lett. 106, 216102 (2011).

[10] T. Trevethan, M. Watkins, and A. L. Shluger, Beilstein J. Nanotechnol. 3, 329 (2012).

[11] R. Smoluchowski, Phys. Rev. 60, 661 (1941).

[12] J. Repp, G. Meyer, F. E. Olsson, and M. Persson, Science 305, 493 (2004).

[13] J. Repp, G. Meyer, S. M. Stojković, A. Gourdon, and C. Joachim, Phys. Rev. Lett. 94, 026803 (2005).

[14] M. Sterrer, T. Risse, U. Martinez-Pozzoni, L. Giordano, M. Heyde, H.-P. Rust, G. Pacchioni, and H.-J. Freund, Phys. Rev. Lett. 98, 096107 (2007).

[15] C. F. Hirjibehedin, C. P. Lutz, and A. J. Heinrich, Science 312, 1021 (2006).

[16] S. Loth, K. von Bergmann, M. Ternes, A. F. Otte, C. P. Lutz, and A. J. Heinrich, Nat. Phys. 6, 340 (2010).

[17] C. F. Hirjibehedin, C.-Y. Lin, A. F. Otte, M. Ternes, C. P. Lutz, B. A. Jones, and A. J. Heinrich, Science 317, 1199 (2007).

[18] T. Choi, C. D. Ruggiero, and J. A. Gupta, Phys. Rev. B 78, 035430 (2008).
[19] F. M. Leibsle, S. S. Dhesi, S. D. Barrett, and A. W. Robinson, Surf. Sci. 317, 309 (1994).

[20] Y. Yoshimoto and S. Tsuneyuki, Surf. Sci. 514, 200 (2002).

[21] A. Soon, L. Wong, B. Delley, and C. Stampfl, Phys. Rev. B 77, 125423 (2008).

[22] F. J. Giessibl, Appl. Phys. Lett. 76, 1470 (2000).

[23] L. Bartels, G. Meyer, and K.-H. Rieder, Appl. Phys. Lett. 71, 213 (1997).

[24] J. E. Sader and S. P. Jarvis, Appl. Phys. Lett. 84, 1801 (2004).

[25] See Supplemental Material at http://link.aps.org/ supplemental/10.1103/PhysRevLett.112.166102 for the calculation of the tip-sample distance for metal tips, an estimation of the error in the calculated tip-sample distance for metal tips, a determination of the tip-sample distance for CO-terminated tips, details for the electrostatic simulation, the raw data of Fig. 2, the frequency shift and force vs distance spectra, and experimental uncertainties in the force measurement.

[26] L. Gross, N. Moll, F. Mohn, A. Curioni, G. Meyer, F. Hanke, and M. Persson, Phys. Rev. Lett. 107, 086101 (2011).

[27] C. J. Chen, Introduction to Scanning Tunneling Microscopy (Oxford University Press, New York, 1993).

[28] J. E. Lennard-Jones and B. M. Dent, Trans. Faraday Soc. 24, 92 (1928).

[29] J. Welker and F. J. Giessibl, Science 336, 444 (2012).

[30] T. Hofmann, F. Pielmeier, and F. J. Giessibl, Phys. Rev. Lett. 112, 066101 (2014).

[31] A. C. Pavão, M. Braga, C. A. Taft, B. L. Hammond, and W. A. Lester, Jr., Phys. Rev. B 43, 6962 (1991).

[32] M. Feng, P. Cabrera-Sanfelix, C. Lin, A. Arnau, D. Sánchez-Portal, J. Zhao, P. M. Echenique, and H. Petek, ACS Nano 5, 8877 (2011).

[33] R. P. Feynman, Phys. Rev. 56, 340 (1939). 